\title{
Effect of Prior Atorvastatin Treatment on the Frequency of Hospital Acquired Pneumonia and Evolution of Biomarkers in Patients with Acute Ischemic Stroke: A Multicenter Prospective Study
}

\author{
Yuetian Yu, ${ }^{1}$ Cheng Zhu, ${ }^{2}$ Chunyan Liu, ${ }^{3}$ and Yuan Gao ${ }^{1}$ \\ ${ }^{1}$ Department of Critical Care Medicine, Renji Hospital, School of Medicine, Shanghai Jiao Tong University, Shanghai 200001, China \\ ${ }^{2}$ Department of Emergency, Ruijin Hospital, School of Medicine, Shanghai Jiao Tong University, Shanghai 200025, China \\ ${ }^{3}$ Department of Emergency, Minhang District Central Hospital, Shanghai 201100, China
}

Correspondence should be addressed to Chunyan Liu; doctorlcy@sina.com and Yuan Gao; gaoyuanzhuren@sina.com

Received 12 July 2016; Revised 23 December 2016; Accepted 4 January 2017; Published 5 March 2017

Academic Editor: Fabrizio Montecucco

Copyright (C) 2017 Yuetian Yu et al. This is an open access article distributed under the Creative Commons Attribution License, which permits unrestricted use, distribution, and reproduction in any medium, provided the original work is properly cited.

Objective. To investigate whether prior treatment of atorvastatin reduces the frequency of hospital acquired pneumonia (HAP). Methods. Totally, 492 patients with acute ischemic stroke and Glasgow Coma Scale $\leq 8$ were enrolled in this study. Subjects were assigned to prior atorvastatin treatment group $(n=268, \mathrm{PG})$ and no prior treatment group $(n=224, \mathrm{NG})$. All the patients were given $20 \mathrm{mg}$ atorvastatin every night during their hospital stay. HAP frequency and 28-day mortality were measured. Levels of inflammatory biomarkers [white blood cell (WBC), procalcitonin (PCT), tumor necrosis factor-alpha (TNF- $\alpha$ ), and interleukin6 (IL-6)] were tested. Results. There was no significant difference in the incidence of HAP between PG and NG $(25.74 \%$ versus. $24.55 \%, p>0.05)$ and 28 -day mortality $(50.72 \%$ versus $58.18 \%, p>0.05)$. However, prior statin treatment did modify the mortality of ventilator associated pneumonia (VAP) $(36.54 \%$ versus $58.14 \%, p=0.041)$ and proved to be a protective factor $(\mathrm{HR}, 0.564 ; 95 \%$ CI, $0.310 \sim 0.825, p=0.038)$. Concentrations of TNF- $\alpha$ and IL-6 in PG VAP cases were lower than those in NG VAP cases $(p<0.01)$. Conclusions. Prior atorvastatin treatment in patients with ischemic stroke was associated with a lower concentration of IL-6 and TNF- $\alpha$ and improved the outcome of VAP. This clinical study has been registered with ChiCTR-ROC-17010633 in Chinese Clinical Trial Registry.

\section{Background}

Hospital acquired pneumonia (HAP) which constitutes a frequent infection in intensive care unit (ICU) patients consumes vast healthcare resources and increases proportionally to the duration of ICU stay. Ventilator associated pneumonia (VAP) is defined as HAP in patients receiving mechanical ventilation $[1,2]$. The incidence of HAP depends on the population studied. For example, in patients with ischemic stroke who are characterized by advanced age, depressed level of consciousness, immune suppression, and long-term bed rest, the incidence of HAP can increase to approximately $40 \%$. It is recognized that one-third to half of all HAP-related deaths are directly attributable to pneumonia [3]. Despite improvements in bundle care prevention, mortality continues to remain high. Therefore, new effective adjunctive therapies are still needed for HAP prevention and treatment.

Statins (3-hydroxy-3-methylglutaryl coenzyme A reductase inhibitors) present anti-inflammatory effects besides their ability to regulate cholesterol composition these years $[4,5]$. Studies have also found statins to possess immunomodulating properties and to be linked to favorable outcomes from sepsis [6].

However, little is known about the mechanisms by which statins counteract inflammation and there is still uncertainty about the risks and benefits of administering statins 
de novo or in continuing statin therapy in patients with infectious diseases [7-9]. It has therefore been deduced that these pleiotropic characteristics of statins might relate to cumulative statins treatment time and their concentration in blood. We hypothesized that statins treatment for a long time might decrease the incidence of HAP and influence the inflammatory response to infection.

\section{Materials and Methods}

The present study is a three-center, two-group, prospective observational cohort trial. All the three teaching hospitals are affiliated to Shanghai Jiaotong University School of Medicine (155 ICU beds in total). The study was approved by the Review Board and Ethics Committee of Shanghai Jiaotong University and informed consent was obtained for all patients, from either the patient or the next of kin. The study took place at the three ICUs from January 2008 to December 2013.

2.1. Study Population. Consecutive sampling was used to recruit critical patients. Patients were eligible for enrolment if they were diagnosed with acute ischemic stroke by magnetic resonance imaging (MRI); were aged between 18 and 80 years; had Glasgow Coma Scale (GCS) $\leq 8$. Patients were excluded from the study if they were moribund or not expected to survive 28 days because of an underlying irreversible medical condition; had active pneumonia when admitted to ICU or had extrapulmonary infection during ICU stay; had a known intolerance to statins or were unable to have enteral administration of statin; had severe liver disease (acute liver failure or chronic liver disease with Child-Pugh classification C); had a serum alanine aminotransferase (ALT) or aspartate aminotransferase (AST) level greater than three times the normal value or a serum creatinine kinase (CK) level greater than five times the upper limit of normal levels; had commenced statin therapy less than 4 weeks before hospital admission or had statin stopped for more than 72 hours before the development of ischemic stroke; were pregnant or if informed consent could not be obtained.

2.2. Study Protocol. All the patients eligible were enrolled in this research during our study period. Depending on previous statin administration, they were classified into prior atorvastatin treatment group (prior use atorvastatin for more than one month before being admitted to ICU, PG) and no prior treatment group (did not use atorvastatin before being admitted to ICU, NG).

All the patients were followed up and were divided by the Oxfordshire Community Stroke Project (OCSP) into four subtypes as total anterior circulation infarcts (TACI), partial anterior circulation infarcts (PACI), posterior circulation infarcts (POCI), and lacunar infarcts (LACI) [10]. All the patients selected were cared for in the ICUs by doctors on duty. Therapeutic schedule was established during ward rounds (three times daily at 8:00, 15:00, and 22:00) by attending doctors or at any emergency moment of the patients. In accordance with the guidelines by the American Heart Association and American Stroke Association [11, 12] and the secondary prevention strategy after ischemic stroke $[13,14]$, all the patients were ordered atorvastatin which is the most commonly prescribed statin in China. Atorvastatin $20 \mathrm{mg}$ was administered every night via enteral feeding tube or per os during stay in ICU for up to 28 days or until death or discharge from the ICU, whichever of these occurred first [15]. A $20 \mathrm{mg}$ dose was chosen (lower end of the dosing range) because of current safety concerns and previous pharmacokinetic study suggesting high plasma levels upon administration [16].

2.3. Clinical Assessment. Baseline assessment included the evaluation of demographic data (age and gender), medical history, stroke subtype, need for invasive positive pressure ventilation (IPPV), ratio of partial oxygen to fraction of inspired oxygen $\left(\mathrm{PaO}_{2} / \mathrm{FiO}_{2}\right)$, acute physiology and chronic health evaluation score (APACHE II), Glasgow Coma Scale (GCS), modified clinical pulmonary infection score (CPIS), lipid concentration, hepatic function, and level of inflammatory biomarkers.

2.4. Diagnosis, Treatment, and Prevention of HAP or VAP. HAP is suspected if the patient has a radiographic infiltrate that is new or progressive, along with clinical findings that suggest infection, which include the onset of fever (temperature $\left.\geq 38.3^{\circ} \mathrm{C}\right)$, leukocytosis $\left(\geq 10 \times 10^{9} / \mathrm{L}\right.$ or $\left.\leq 4 \times 10^{9} / \mathrm{L}\right)$, purulent sputum, and decline in oxygenation. In addition, a positive tracheal aspirate quantitative culture $\left(\geq 10^{5}\right.$ colonyforming units $/ \mathrm{mL}$ ) or a positive bronchoalveolar lavage fluid (BALF) culture ( $\geq 10^{4}$ colony-forming units $\left./ \mathrm{mL}\right)$ is required to confirm the diagnosis of HAP. When this definition has been applied to mechanically ventilated patients, then VAP is suspected $[17,18]$.

The protocol for HAP or VAP treatment and prevention followed standard protocols in all institutions also based on accepted guidelines. Care bundles for preventing HAP or VAP were also used including daily assessments of sedation, daily oral hygiene, the use of a semirecumbent position with the head of the bed elevated to at least $30^{\circ}$, peptic ulcer prophylaxis, and venous thromboembolism prophylaxis [1719].

One month before the start of this study, a standardized sampling, processing, analysis, and statistics procedure was set by 6 investigators from these centers after 3 days' learning and discussion. All of the centers carefully followed this procedure during the study time. There was no significant difference in compliance among the three centers.

2.5. Measurement of Blood and Microbiology. Blood samples were collected for the measurement of inflammatory biomarkers at baseline and at the first, third, fifth, seventh, and tenth day after the clinical diagnosis of HAP or VAP. ALT, AST, and CK were tested on Monday and Thursday every week. All the blood samples were separated by centrifugation (TDL-60C, 6000 revolutions per minute, China). Commercially available ELISA assays were used for detecting inflammatory biomarkers. White blood cell (WBC) and differential blood counts were determined using a Coulter STKS clinical analyzer (Coulter Electronics Inc., Miami, FL, USA). Procalcitonin (PCT) levels were obtained 


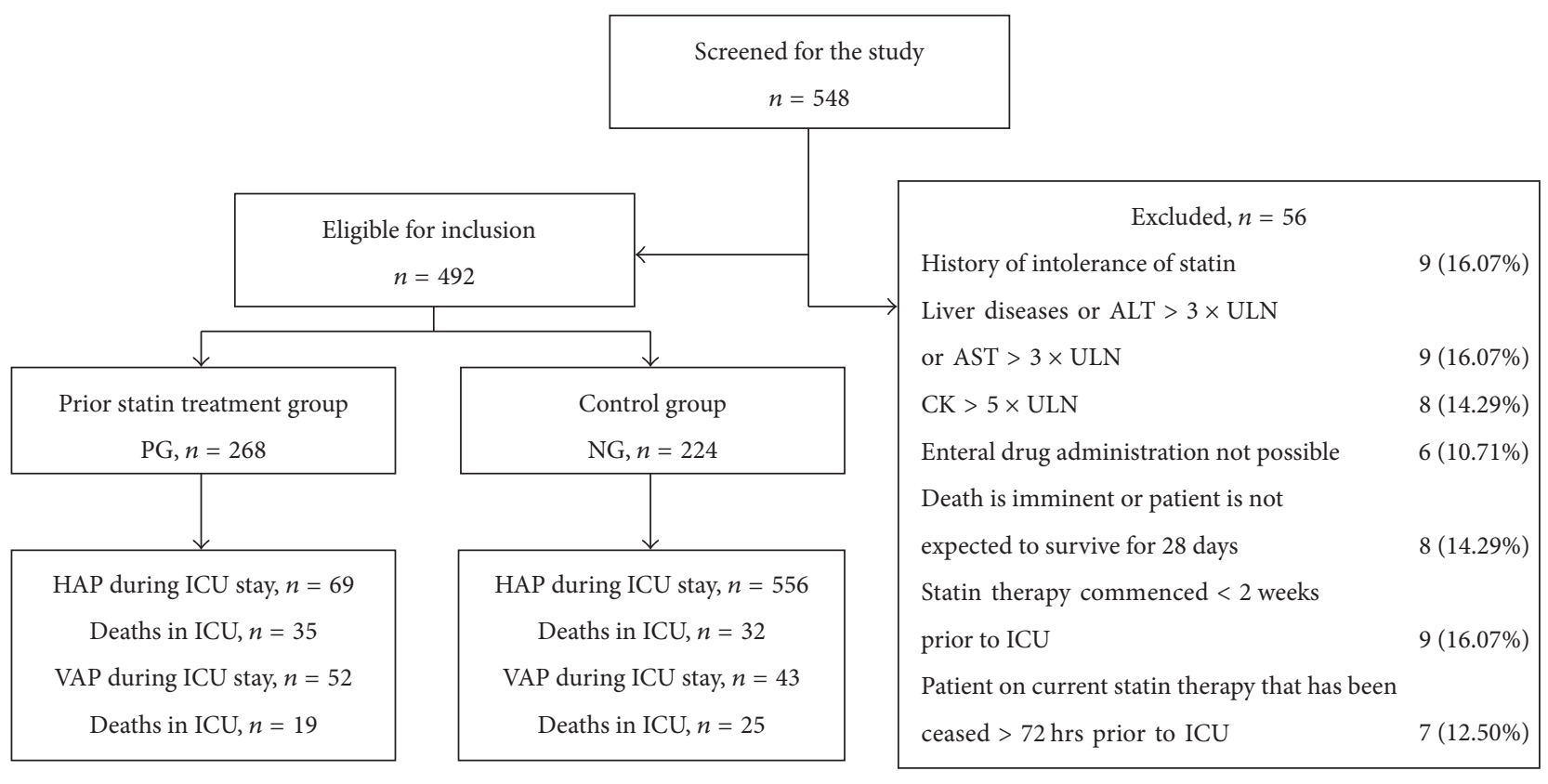

FIGURE 1: Flowchart of the study. ULN: upper limit of normal.

using the enzyme-linked fluorescent assay (ELFA) technique. This assay combines a one-step immunoassay sandwich method with a final automatic fluorescent detection by a VIDAS instrument (VIDAS BRAHMS PCT, bioMérieux, Lyon, France). The measurement range of this instrument was set to $0.05 \sim 200 \mathrm{ng} / \mathrm{mL}$. Serum concentrations of interleukin6 (IL-6) and tumor necrosis factor-alpha (TNF- $\alpha$ ) were measured using a CBA kit (BDTM Cytometric Bead ArrayHuman Inflammatory cytokine kit) The detection limit of the methods for the TNF- $\alpha$ was $20 \mathrm{pg} / \mathrm{mL}$ and $1.88 \sim 120 \mathrm{ng} / \mathrm{mL}$ for IL-6.

To determine which bacterial species was responsible for pneumonia, bronchial secretions or BALF were sampled at the baseline and at the first, third, fifth, seventh, and tenth day after clinical diagnosis of HAP or VAP. The diagnostic flexible bronchoscopy guideline of British Thoracic Society [20] was followed. Microbiology assessment included identification and quantitative assessment of the microbial agent and evaluation of its in vitro resistance to antibiotics.

2.6. Outcomes. We primarily assessed the morbidity of HAP or VAP and mortality over a 28-day treatment in ICU or at the end of ICU treatment. In addition, we assessed the evolution of inflammatory biomarkers (WBC; PCT; TNF- $\alpha$; IL-6). Adverse events related to atorvastatin treatment (CK abnormal changes, hepatic enzyme dysfunction) were also documented.

2.7. Statistical Analysis. Statistical analysis was performed by using SPSS version 19.0 (IBM for Windows). Data were initially assessed for normality and log transformed where appropriate. Data between the PG and NG were compared using Chi-square test for equal proportion or Fisher exact test where numbers were small with results presented as percentages $(n)$. Continuously normally distributed variables were compared using Student's $t$-test and presented as means (standard deviations), whereas nonnormally distributed data was compared using Wilcoxon rank-sum test and reported as medians (interquartile range). Differences in changes of inflammatory biomarkers (dependent variables) with time were analyzed by using a general linear model for repeated measures with Bonferroni's adjustment. Logistic regression models were used for multivariate analysis, in which we included all variables found to be related to outcomes in univariate analysis. The probability of ICU survival was assessed with Kaplan-Meier survival analysis, and comparisons between groups were performed using the log-rank (Mantel-Cox) test. Cox proportional hazards regression analysis was also used to examine the effect of multiple risk factors on mortality. Factors associated significantly $(p<0.05)$ with mortality in univariate analyses were entered in multivariable analyses. The statistical tests performed were two-sided. All analysis was performed on an intention-to-treat basis and a two-sided $p<0.05$ was considered to be statistically significant. Figures were drawn by using GraphPad prism version 5.0 (GraphPad Software for Windows).

\section{Results}

A total of 548 patients were screened and 492 eligible patients were recruited for the study (102 patients were enrolled in Renji Hospital, 217 patients were enrolled in Ruijin Hospital, and 173 patients were enrolled in Minhang District Central Hospital). A total of 268 patients were assigned to PG, and 224 patients to NG. Fifty-six patients who met the exclusion criteria were excluded from the study (Figure 1). 
TABLE 1: Baseline characteristics of all 492 patients.

\begin{tabular}{|c|c|c|c|}
\hline Variable & $\mathrm{PG}(n=268)$ & $\mathrm{NG}(n=224)$ & $p$ \\
\hline Male gender, $n(\%)$ & $130(48.51)$ & $108(48.21)$ & 1.000 \\
\hline Age, yrs & $68.34 \pm 7.73$ & $67.13 \pm 7.82$ & 0.085 \\
\hline \multicolumn{4}{|l|}{ Previous medical illness } \\
\hline $\begin{array}{l}\text { Cardiovascular } \\
\text { disease, } n(\%)\end{array}$ & $175(65.30)$ & $123(54.91)$ & 0.019 \\
\hline $\begin{array}{l}\text { Cerebrovascular } \\
\text { disease, } n(\%)\end{array}$ & $110(41.04)$ & $70(31.25)$ & 0.025 \\
\hline \multicolumn{4}{|l|}{ Position of stroke } \\
\hline TACI, $n(\%)$ & $64(23.88)$ & $49(21.88)$ & \multirow{4}{*}{0.838} \\
\hline PACI, $n(\%)$ & $51(19.03)$ & $46(20.54)$ & \\
\hline POCI, $n(\%)$ & $68(25.37)$ & $52(23.21)$ & \\
\hline LACI, $n(\%)$ & $85(31.72)$ & $77(34.37)$ & \\
\hline IPPV, $n(\%)$ & $132(49.25)$ & $101(49.05)$ & 0.366 \\
\hline Modified CPIS & $3.35 \pm 1.22$ & $3.58 \pm 1.72$ & 0.084 \\
\hline GCS & $5.32 \pm 1.34$ & $5.08 \pm 1.74$ & 0.085 \\
\hline APACHE II & $17.56 \pm 3.43$ & $18.24 \pm 4.32$ & 0.052 \\
\hline $\mathrm{PaO}_{2} / \mathrm{FiO}_{2}(\mathrm{mmHg})$ & $332.45 \pm 43.55$ & $340.47 \pm 48.58$ & 0.054 \\
\hline Cholesterol (mmol/L) & $4.58 \pm 0.76$ & $5.13 \pm 1.10$ & 0.001 \\
\hline $\mathrm{LDL}(\mathrm{mmol} / \mathrm{L})$ & $3.13 \pm 0.45$ & $3.19 \pm 0.34$ & 0.001 \\
\hline $\operatorname{ALT}(\mathrm{U} / \mathrm{L})$ & $58.33 \pm 5.45$ & $57.65 \pm 5.52$ & 0.170 \\
\hline $\operatorname{AST}(\mathrm{U} / \mathrm{L})$ & $23.09 \pm 3.15$ & $23.57 \pm 2.45$ & 0.064 \\
\hline CK (ng/mL) & $45.33 \pm 3.56$ & $46.01 \pm 4.89$ & 0.076 \\
\hline WBC $\left(10^{9} / \mathrm{L}\right)$ & $8.72 \pm 1.43$ & $8.55 \pm 0.93$ & 0.127 \\
\hline PCT (ng/mL) & $0.03 \pm 0.01$ & $0.03 \pm 0.01$ & 1.000 \\
\hline TNF- $\alpha(n g / L)$ & $0.55 \pm 0.09$ & $0.56 \pm 0.08$ & 0.198 \\
\hline IL-6 (ng/mL) & $28.54 \pm 5.87$ & $27.67 \pm 5.98$ & 0.105 \\
\hline
\end{tabular}

3.1. Baseline. Four hundred and ninety-two eligible patients were recruited in the final analysis. The baseline characteristics of all 492 patients are presented at Table 1 . The demographic data, position of stroke, $\mathrm{PaO}_{2} / \mathrm{FiO}_{2}$, APACHE II, GCS, modified CPIS, hepatic function, and levels of inflammatory biomarkers were not significantly different between PG and NG. As expected, patients in PG had significantly higher rates of ischemic heart disease, ischemic cerebral disease, and a lower level of lipid because of long time treatment of statins.

3.2. Primary Outcomes. One hundred and twenty-four patients $(25.20 \%)$ were diagnosed with HAP during the 28day treatment in ICU. Sixty-nine patients (25.74\%) were from PG and 55 (24.55\%) from NG. Thirty-five patients (50.72\%) in PG and 32 patients (58.18\%) in NG died during the 28-day treatment period in ICU. Although there was an indication of a trend toward a higher mortality in NG, there was no statistical significant difference in 28-day ICU mortality or the incidence of HAP between the two groups $(p>0.05)$.

The ICU treatment duration before HAP diagnosis was 13.15 days in PG and 12.68 days in NG. The incidence of septic shock was $30.43 \%$ in PG and $34.55 \%$ in NG. Body temperature, WBC, PCT, TNF- $\alpha$, and IL- 6 were not different
TABLE 2: Characteristics at the day of HAP diagnosis.

\begin{tabular}{lccc}
\hline Variable & PG $(n=69)$ & NG $(n=55)$ & $p$ \\
\hline MDR cases, $n(\%)$ & $32(46.38)$ & $27(49.09)$ & 0.857 \\
Septic shock, $n(\%)$ & $21(30.43)$ & $19(34.55)$ & 0.700 \\
ICU stay before HAP, (days) & $13.15 \pm 4.32$ & $12.68 \pm 5.01$ & 0.576 \\
Temperature $\left({ }^{\circ} \mathrm{C}\right)$ & $38.03 \pm 0.45$ & $38.18 \pm 0.53$ & 0.091 \\
WBC $\left(10^{9} / \mathrm{L}\right)$ & $15.73 \pm 3.67$ & $16.04 \pm 3.05$ & 0.616 \\
PCT $(\mathrm{ng} / \mathrm{mL})$ & $1.34 \pm 0.23$ & $1.25 \pm 0.32$ & 0.071 \\
TNF- $\alpha(\mathrm{ng} / \mathrm{L})$ & $2.87 \pm 0.34$ & $2.76 \pm 0.46$ & 0.129 \\
IL-6 $(\mathrm{ng} / \mathrm{mL})$ & $85.56 \pm 7.78$ & $83.57 \pm 6.87$ & 0.139 \\
\hline
\end{tabular}

TABLE 3: Characteristics of IPPV patients at the day of VAP diagnosis.

\begin{tabular}{lccc}
\hline Variable & PG $(n=52)$ & NG $(n=43)$ & $p$ \\
\hline Position of stroke & & & \\
$\quad$ TACI, $n(\%)$ & $9(17.31)$ & $6(13.95)$ & 0.780 \\
$\quad$ POCI, $n(\%)$ & $43(82.69)$ & $37(86.05)$ & \\
Modified CPIS & $8.11 \pm 2.75$ & $8.23 \pm 2.56$ & 0.828 \\
GCS & $4.12 \pm 0.87$ & $4.02 \pm 0.73$ & 0.551 \\
APACHE II & $22.05 \pm 3.39$ & $21.87 \pm 3.47$ & 0.799 \\
\hline
\end{tabular}

between the PG and NG at the day of diagnosed HAP ( $p>$ 0.05 , Table 2).

In the HAP patients, a total of 11 different species of microorganisms were isolated. The most frequently isolated microorganisms were Pseudomonas aeruginosa (33.2\%) and Klebsiella species (28.5\%). In 19 patients, more than one pathogen was detected. Univariate analysis revealed no significant differences between the two groups for the types of pathogens.

Two hundred and thirty-three (47.35\%) patients enrolled in the study needed IPPV therapy for more than $48 \mathrm{~h}$. Ninetyfive patients $(40.77 \%)$ with IPPV therapy presented with VAP during the 28-day treatment period in the ICU. Subtypes of stroke, modified CPIS, GCS, and APACHE II of the patients with VAP were presented in Table 3 . The variables were similar $(p>0.05)$. The incidence of VAP was not significantly different between the two groups (39.39\% versus $42.57 \%$, $p=$ 0.581 ). However, prior statin therapy did modify the outcome of VAP (36.54\% versus $58.14 \%, p=0.041$, Figure 2 ). Cox regression analysis for the effect of multiple factors on VAP mortality after 28-day ICU treatment is given in Table 4. Prior statin treatment proved to be the one protective factor (HR, 0.564; 95\% CI, 0.310 0.825, $p=0.038$, Table 4).

3.3. Secondary Outcomes. Inflammatory biomarkers were collected and measured at the first, third, fifth, seventh, and tenth day after the clinical diagnosis of VAP. WBC and PCT assessed during the study were not significantly different between PG and NG in serum ( $p>0.05)$. However, the concentrations of TNF- $\alpha$ and IL-6 in PG VAP cases were lower than those in NG VAP cases in serum $(p<0.01$, Table 5, Figure 3). 

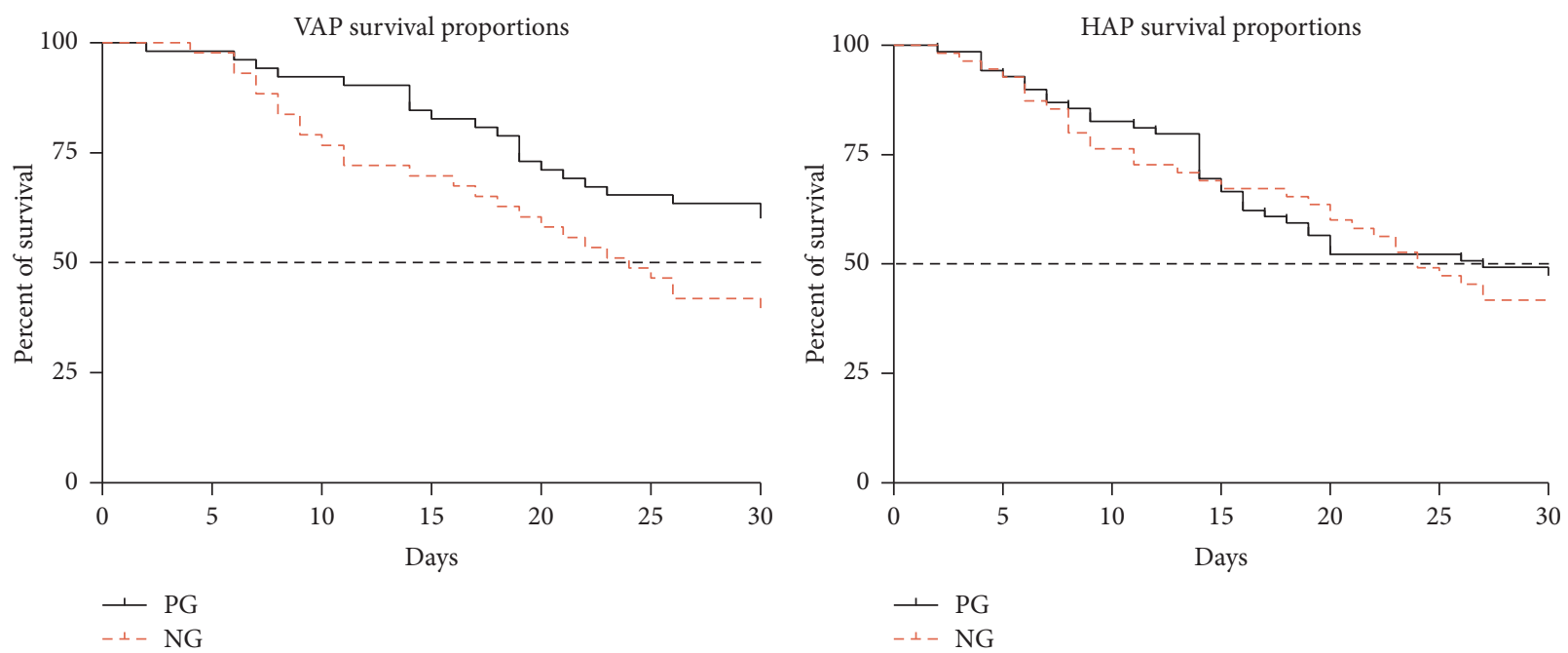

FIgURE 2: Probability of survival of the patients with HAP or VAP during 28-day ICU treatment. The dashed black line refers to $50 \%$ of survival (median survival reference line).

TABLE 4: Cox regression analysis for the effect of multiple factors on mortality of patients with HAP.

\begin{tabular}{lccc}
\hline Variable & HR & $95 \%$ CI & $p$ \\
\hline Prior statin treatment & 0.564 & $0.310 \sim 0.825$ & 0.038 \\
Age & 2.683 & $1.304 \sim 4.668$ & 0.041 \\
Gender (male) & 1.083 & $0.835 \sim 1.476$ & 0.882 \\
Position of stroke & 4.494 & $1.086 \sim 9.598$ & 0.032 \\
CPIS & 3.884 & $2.883 \sim 7.936$ & 0.042 \\
GCS & 2.674 & $1.983 \sim 8.836$ & 0.036 \\
APACHE II & 2.563 & $1.376 \sim 8.763$ & 0.042 \\
Cholesterol & 1.542 & $0.732 \sim 6.424$ & 0.453 \\
LDL & 1.145 & $0.832 \sim 5.324$ & 0.464 \\
Previous cardiovascular disease & 1.185 & $0.860 \sim 4.562$ & 0.645 \\
Previous cerebrovascular disease & 1.874 & $0.874 \sim 7.5124$ & 0.644 \\
\hline
\end{tabular}

3.4. Adverse Effects. Atorvastatin administered at $20 \mathrm{mg}$ every night was relatively safe in the study group. Serious adverse events as defined herein did not occur in the present study. Slight elevations in CK occurred in both PG and NG. Hepatic function was similar between the two groups. Myopathy was not diagnosed.

\section{Discussion}

4.1. Key Findings. In this prospective observational study, we found that therapy with statins for at least one month before the onset of acute ischemic stroke was probably associated with a decreased mortality of VAP during 28-day ICU treatment. Concentrations of TNF- $\alpha$ and IL-6 in PG VAP cases were lower than those in NG VAP cases in serum.

4.2. Relationship with Previous Studies. The findings of our study suggest that prior statins treatment may be useful as an adjunctive therapy in critically ill patients and notably improves the outcome. In this respect, our results support
TABLE 5: Inflammatory markers at the day of VAP diagnosis and 10 days of treatment.

\begin{tabular}{lccc}
\hline Variable & PG & NG & $p$ \\
\hline Day of VAP diagnosis & $n=52$ & $n=43$ & \\
WBC $\left(10^{9} / \mathrm{L}\right)$ & $16.85 \pm 4.65$ & $17.46 \pm 4.78$ & 0.531 \\
PCT $(\mathrm{ng} / \mathrm{mL})$ & $2.03 \pm 0.34$ & $1.98 \pm 0.41$ & 0.517 \\
TNF- $\alpha(\mathrm{ng} / \mathrm{L})$ & $3.88 \pm 0.57$ & $4.71 \pm 0.42$ & 0.001 \\
IL-6 $(\mathrm{ng} / \mathrm{mL})$ & $80.34 \pm 5.45$ & $83.25 \pm 5.28$ & 0.010 \\
10 days of treatment & $n=49$ & $n=38$ & \\
WBC $\left(10^{9} / \mathrm{L}\right)$ & $12.02 \pm 2.45$ & $11.47 \pm 2.56$ & 0.311 \\
PCT $(\mathrm{ng} / \mathrm{mL})$ & $0.51 \pm 0.12$ & $0.53 \pm 0.13$ & 0.459 \\
TNF- $\alpha(\mathrm{ng} / \mathrm{L})$ & $3.76 \pm 0.68$ & $5.02 \pm 0.47$ & 0.001 \\
IL-6 $(\mathrm{ng} / \mathrm{mL})$ & $43.54 \pm 4.45$ & $57.25 \pm 5.68$ & 0.001 \\
\hline
\end{tabular}

the findings of previous studies which suggest that statins may favorably affect critically ill patients or animals [2124]. Although previous studies demonstrated a beneficial effect of prior statin use on the outcome of patients with pneumonia, a large prospective study came to a different conclusion which suggested a potentially harmful risk of statins $[9,25]$. In our opinion, it should be pointed out that those previous studies neither classified the patients who had already been on chronic statin treatment nor considered the plasma statins concentration. The subjects of our study were carefully selected to avoid misinterpretation of results.

One previous study provides evidence that the addition of oral pravastatin in the usual treatment regime of ICU patients might have significant effects on mortality of more severely ill patients (APACHE II $\geq 15$ ) [21]. A majority of the patients included in our study were more severe than those in the previous study. The average of APACHE II was higher than 15 , which means that the assumed mortality of our patients was higher than $25 \%$. Our data suggested that the probability of survival during the 28 -day treatment period was decreased 

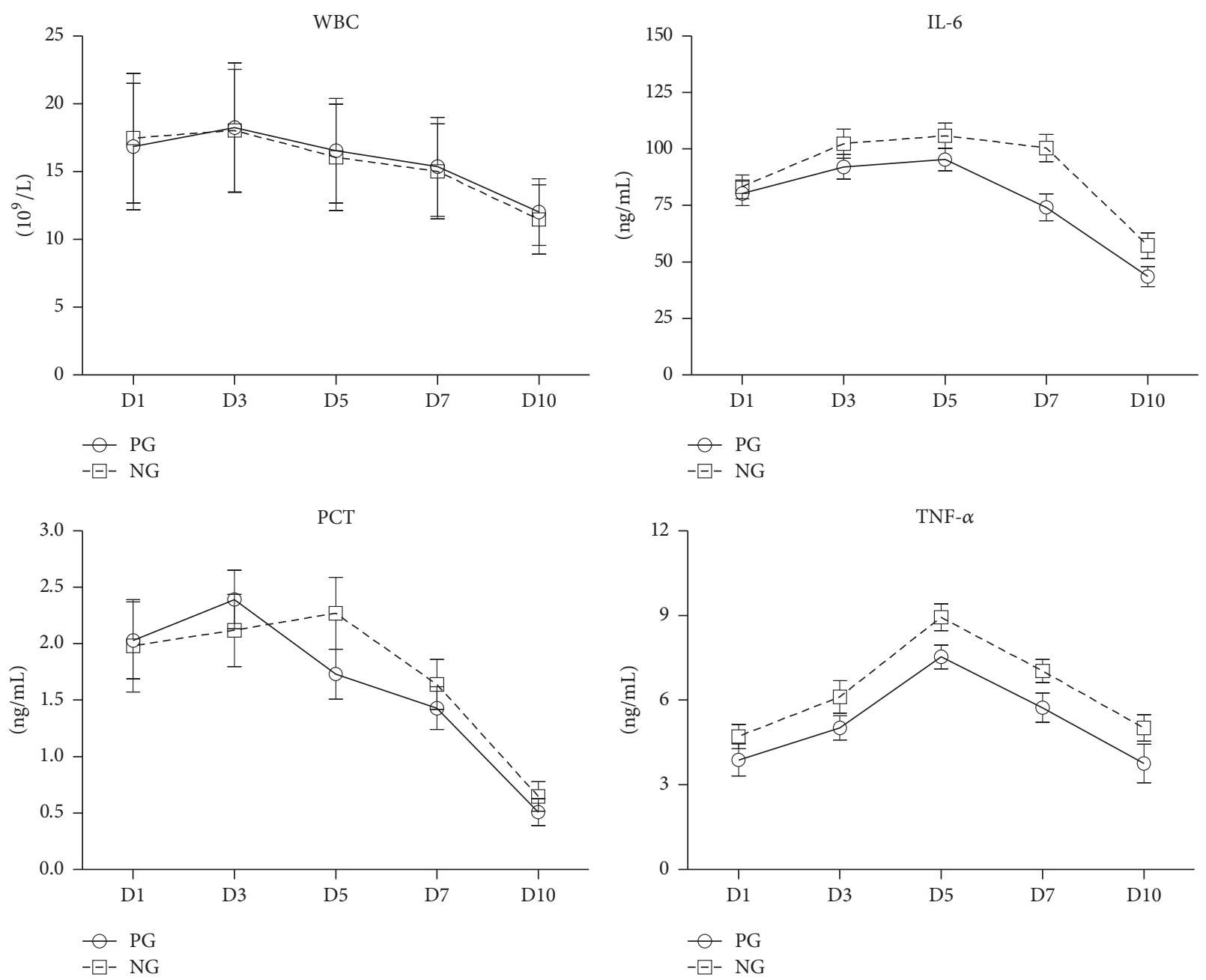

Figure 3: Evolution of WBC, PCT, TNF- $\alpha$, and IL-6 in blood serum in VAP cases.

in PG compared to that of NG $(p=0.041)$. These findings suggest that statins may be useful as an adjunctive therapy in critically ill patients. In this respect, our results support the findings of previous studies which suggested that statins might favorably affect the course of critically ill patients [21].

4.3. Study Significance. In the present study, we evaluated the effect of prior treatment of atorvastatin on HAP or VAP frequency in ischemic stroke patients. To the best of our knowledge, this is the first study which provides evidence supporting the idea that prior statins treatment might affect the frequency of HAP or VAP in patients with ischemic stroke and decrease the inflammatory response. Our findings suggest that although prior treatment with atorvastatin did not significantly affect the incidence of HAP or VAP, it did modify the outcome of VAP. Additionally, continued use of atorvastatin therapy in prior statin users was associated with lower levels of TNF- $\alpha$ and IL- 6 in serum. This suggests a potential benefit of continued administration or de novo use in infectious diseases. We assume that this might be explained by potential "anti-inflammatory" or "anti-infectious" capability of statins, which may reduce the inflammatory burden of the most severe forms of infectious illness such as VAP $[2,26]$.

In this investigation, we mainly followed the evolution of clinical and biological inflammatory biomarkers that are commonly used in ICU and we have not assessed specific pathways that have been reported to be affected by statins. The levels of TNF- $\alpha$ and IL- 6 were decreased by atorvastatin treatment in PG patients with VAP. It should also be noted here that the properties of statins act through down- or upregulation of cytokines, modification of the function of leukocytes and lymphocytes, and direct inhibition of major histocompatibility complex II [27]. Furthermore, it was previously reported that statins can cause an increase in hemeoxygenase activity and may reduce the oxidative burden in tissues during sepsis [28]. Therefore, statins may modify the inflammatory cascade and inhibit the progression of septic conditions. TNF- $\alpha$ and IL- 6 are pleiotropic cytokines and central mediators of the acute-phase response. They are produced by vascular endothelial cells, smooth muscle cells, and leukocytes with a broad range of effects on diverse immune cells $[29,30]$. Atorvastatin is anti-inflammatory and acts by downregulating cytokines in the endothelium and 
leukocytes. In this study, atorvastatin decreased the concentrations of TNF- $\alpha$ and IL- 6 in serum. However, these antiinflammatory effects may need sustained statin treatment so as to maintain a relatively high plasma concentration of statin.

4.4. Study Limitations. We acknowledge that there are limitations to this study. We included a homogeneous population of ischemic stroke which might minimize the heterogeneity and avoided misinterpreting the study result. Atorvastatin was the only type of statins in our study and no placebo arm was established. We also could not provide the factors associated with VAP mortality. So the application to routine clinical practice of other statins (simvastatin, pravastatin, rosuvastatin, etc.) remains uncertain. Whether the prior treatment of atorvastatin in general may translate into clinical benefits outside this setting still deserves further explorations.

\section{Conclusion}

In conclusion, the present investigation is the first prospective study that assessed the impact of statins in patients with ischemic stroke in their usual treatment. Our findings suggest that prior atorvastatin treatment in ICU may act to decrease the concentrations of TNF- $\alpha$ and IL- 6 . In addition, it may have a beneficial role in the outcome of VAP.

\section{Abbreviations}

ALT: $\quad$ Alanine aminotransferase

APACHE II: Acute physiology and chronic health evaluation II

AST: $\quad$ Aspartate aminotransferase

BALF: Bronchoalveolar lavage fluid

CK: $\quad$ Creatinine kinase

CPIS: $\quad$ Clinical pulmonary infection score

ELISA: $\quad$ Enzyme-Linked Immunosorbent Assay

GCS: $\quad$ Glasgow Coma Scale

HAP: Hospital acquired pneumonia

ICU: Intensive care unit

IL-6: Interleukin-6

IPPV: Invasive positive pressure ventilation

LACI: Lacunar infarcts

LDL: $\quad$ Low density lipoprotein

MRI: $\quad$ Magnetic resonance imaging

OCSP: $\quad$ Oxfordshire Community Stroke Project

PACI: Partial anterior circulation infarcts

PCT: $\quad$ Procalcitonin

POCI: $\quad$ Posterior circulation infarcts

TACI: Total anterior circulation infarcts

TNF- $\alpha$ : Tumor necrosis factor-alpha

VAP: Ventilator associated pneumonia

WBC: White blood cell.

\section{Competing Interests}

The authors have declared that no competing interests exist.

\section{Authors' Contributions}

Yuetian Yu and Cheng Zhu contributed equally to this work.

\section{References}

[1] A. Torres, S. Ewig, H. Lode, and J. Carlet, "Defining, treating and preventing hospital acquired pneumonia: European perspective," Intensive Care Medicine, vol. 35, no. 1, pp. 9-29, 2009.

[2] N. Anand and M. H. Kollef, "The alphabet soup of pneumonia: CAP, HAP, HCAP, NHAP, and VAP," Seminars in Respiratory and Critical Care Medicine, vol. 30, no. 1, pp. 3-9, 2009.

[3] M. J. Richards, J. R. Edwards, D. H. Culver, and R. P. Gaynes, "Nosocomial infections in medical intensive care units in the United States," Critical Care Medicine, vol. 27, no. 5, pp. 887-892, 1999.

[4] W. A. Van Klei and W. F. Buhre, "Anti-inflammatory effects of perioperative statin therapy," Canadian Journal of Anesthesia, vol. 59, no. 6, pp. 516-521, 2012.

[5] M. K. Jain and P. M. Ridker, "Anti-inflammatory effects of statins: clinical evidence and basic mechanisms," Nature Reviews Drug Discovery, vol. 4, no. 12, pp. 977-987, 2005.

[6] Y. Almog, A. Shefer, V. Novack et al., "Prior statin therapy is associated with a decreased rate of severe sepsis," Circulation, vol. 110, no. 7, pp. 880-885, 2004.

[7] R. G. Schlienger, D. S. Fedson, S. S. Jick, H. Jick, and C. R. Meier, "Statins and the risk of pneumonia: A Population-based, Nested Case-control Study," Pharmacotherapy, vol. 27, no. 3, pp. 325332, 2007.

[8] E. M. W. van de Garde, E. Hak, P. C. Souverein, A. W. Hoes, J. M. M. van den Bosch, and H. G. M. Leufkens, "Statin treatment and reduced risk of pneumonia in patients with diabetes," Thorax, vol. 61, no. 11, pp. 957-961, 2006.

[9] S. R. Majumdar, F. A. McAlister, D. T. Eurich, R. S. Padwal, and T. J. Marrie, "Statins and outcomes in patients admitted to hospital with community acquired pneumonia: Population Based Prospective Cohort Study," British Medical Journal, vol. 333, no. 7576, pp. 999-1001, 2006.

[10] J. Bamford, P. Sandercock, M. Dennis, C. Warlow, and J. Burn, "Classification and natural history of clinically identifiable subtypes of cerebral infarction," The Lancet, vol. 337, no. 8756, pp. 1521-1526, 1991.

[11] K. L. Furie, S. E. Kasner, R. J. Adams et al., "Guidelines for the prevention of stroke in patients with stroke or transient ischemic attack: a guideline for healthcare professionals from the American Heart Association/American Stroke Association," Stroke, vol. 42, no. 1, pp. 227-276, 2011.

[12] E. C. Jauch, J. L. Saver, H. P. Adams et al., "Guidelines for the early management of patients with acute ischemic stroke: a guideline for healthcare professionals from the American Heart Association/American Stroke Association," Stroke, vol. 44, no. 3, pp. 870-947, 2013.

[13] S. C. Fagan, "Urgent need for secondary stroke prevention after transient ischemic attack," Consultant Pharmacist, vol. 23, no. 2, pp. 131-140, 2008.

[14] S. M. Davis and G. A. Donnan, "Clinical practice. Secondary prevention after ischemic stroke or transient ischemic attack," The New England Journal of Medicine, vol. 366, no. 20, pp. 19141922,2012

[15] R. Ji, G. Liu, H. Shen et al., "Persistence of secondary prevention medications after acute ischemic stroke or transient ischemic attack in Chinese population: data from China National Stroke Registry," Neurological Research, vol. 35, no. 1, pp. 29-36, 2013.

[16] P. S. Kruger, N. M. Freir, B. Venkatesh, T. A. Robertson, M. S. Roberts, and M. Jones, "A preliminary study of atorvastatin 
plasma concentrations in critically ill patients with sepsis," Intensive Care Medicine, vol. 35, no. 4, pp. 717-721, 2009.

[17] American Thoracic Society and Infectious Diseases Society of America, "Guidelines for the management of adults with hospital-acquired, ventilator-associated, and healthcare-associated pneumonia," American Journal of Respiratory and Critical Care Medicine, vol. 171, no. 4, pp. 388-416, 2005.

[18] J. Muscedere, P. Dodek, S. Keenan, R. Fowler, D. Cook, and D. Heyland, "Comprehensive evidence-based clinical practice guidelines for ventilator-associated pneumonia: diagnosis and treatment," Journal of Critical Care, vol. 23, no. 1, pp. 138-147, 2008.

[19] J. Muscedere, P. Dodek, S. Keenan, R. Fowler, D. Cook, and D. Heyland, "Comprehensive evidence-based clinical practice guidelines for ventilator-associated pneumonia: prevention," Journal of Critical Care, vol. 23, no. 1, pp. 126-137, 2008.

[20] British Thoracic Society Bronchoscopy Guidelines Committee, "British Thoracic Society guidelines on diagnostic flexible bronchoscopy," Thorax, vol. 56, supplement 1, pp. i1-i21, 2001.

[21] D. Makris, E. Manoulakas, A. Komnos et al., "Effect of pravastatin on the frequency of ventilator-associated pneumonia and on intensive care unit mortality: open-label, randomized study," Critical Care Medicine, vol. 39, no. 11, pp. 2440-2446, 2011.

[22] A. Rezaie-Majd, T. Maca, R. A. Bucek et al., "Simvastatin reduces expression of cytokines interleukin-6, interleukin-8, and monocyte chemoattractant protein-1 in circulating monocytes from hypercholesterolemic patients," Arteriosclerosis, Thrombosis, and Vascular Biology, vol. 22, no. 7, pp. 1194-1199, 2002.

[23] M. Z. Chaudhry, J. H. Wang, S. Blankson, and H. P. Redmond, "Statin (cerivastatin) protects mice against sepsis-related death via reduced proinflammatory cytokines and enhanced bacterial clearance," Surgical Infections, vol. 9, no. 2, pp. 183-194, 2008.

[24] P. Kruger, M. Bailey, R. Bellomo et al., "A multicenter randomized trial of atorvastatin therapy in intensive care patients with severe sepsis," American Journal of Respiratory and Critical Care Medicine, vol. 187, no. 7, pp. 743-750, 2013.

[25] P. S. Kruger, M. L. Harward, M. A. Jones et al., "Continuation of statin therapy in patients with presumed infection: a randomized controlled trial," American Journal of Respiratory and Critical Care Medicine, vol. 183, no. 6, pp. 774-781, 2011.

[26] M. Nguile-Makao, J.-R. Zahar, A. Français et al., "Attributable mortality of ventilator-associated pneumonia: respective impact of main characteristics at ICU admission and VAP onset using conditional logistic regression and multi-state models," Intensive Care Medicine, vol. 36, no. 5, pp. 781-789, 2010.

[27] M. B. Fessler, S. K. Young, S. Jeyaseelan et al., "A role for hydroxy-methylglutaryl coenzyme A reductase in pulmonary inflammation and host defense," American Journal of Respiratory and Critical Care Medicine, vol. 171, no. 6, pp. 606-615, 2005.

[28] T. R. Craig, M. J. Duffy, M. Shyamsundar et al., "A randomized clinical trial of hydroxymethylglutaryl- coenzyme a reductase inhibition for acute lung injury (The HARP Study)," American Journal of Respiratory and Critical Care Medicine, vol. 183, no. 5, pp. 620-626, 2011.

[29] J. Van Snick, "Interleukin-6: an overview, Annual Review of Immunology, vol. 8, pp. 253-278, 1990.

[30] H. Wang, K. Zhang, L. Zhao, J. Tang, L. Gao, and Z. Wei, "Anti-inflammatory effects of vinpocetine on the functional expression of nuclear factor-kappa B and tumor necrosis factoralpha in a rat model of cerebral ischemia-reperfusion injury," Neuroscience Letters, vol. 566, pp. 247-251, 2014. 


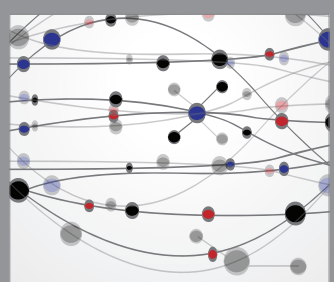

The Scientific World Journal
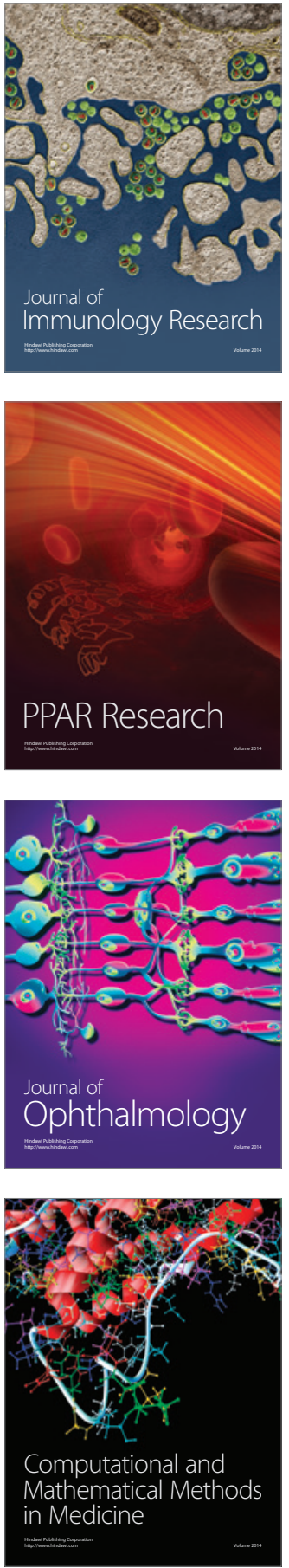

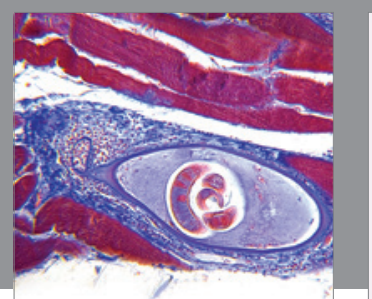

Gastroenterology Research and Practice
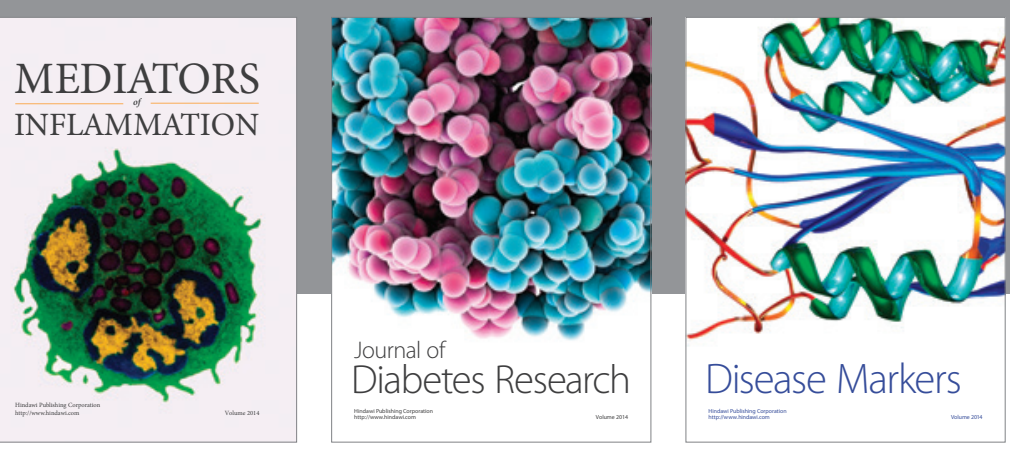

Disease Markers

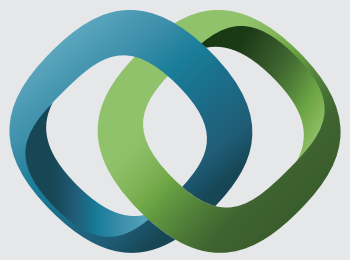

\section{Hindawi}

Submit your manuscripts at

https://www.hindawi.com
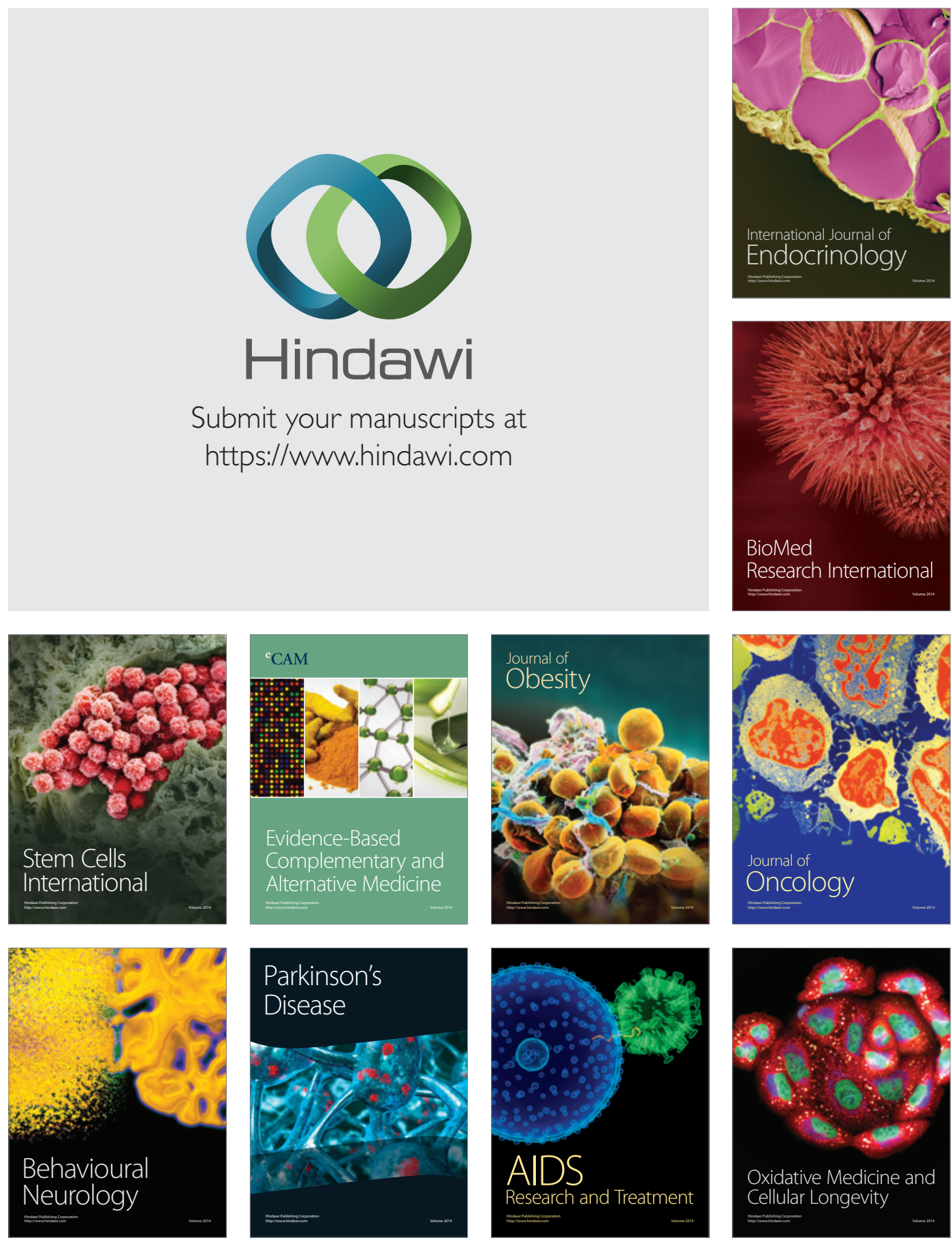